\title{
Lower Bounds for the Numbers of Antipodal Pairs and Strictly Antipodal Pairs of Vertices in a Convex Polytope
}

\author{
Mahn Hung Nguyên and Valeriu Soltan \\ Mathematical Institute of the Academy of Sciences, \\ Academic str. 5, Kishinev 277028, Republica Moldova
}

\begin{abstract}
The greatest lower bounds for the numbers of antipodal pairs and strictly antipodal pairs of vertices in a convex $d$-polytope with $n$ vertices are determined.
\end{abstract}

\section{Introduction}

Let $X$ be a finite set of points in $d$-dimensional Euclidean space $E^{d}$. Following Klee [4], points $x, z \in X$ are called antipodal provided there are two (distinct) parallel hyperplanes $H_{x}, H_{z}$ supporting the polytope $P=\operatorname{conv} X$ such that $x \in H_{x}$ and $z \in H_{z}$. If, additionally, $P \cap H_{x}=\{x\}$ and $P \cap H_{z}=\{x\}$, then the points $x, z$ are called strictly antipodal (see [3]).

Klee [4] posed the problem of determining the maximum number $m$ of points on the surface of a convex body in $E^{d}$, every pair of which is antipodal. Danzer and Grünbaum [1] solved this problem showing that $m \leq 2^{d}$ with $m=2^{d}$ if and only if the points are the vertices of a $d$-parallelotope. Grunbaum [3] proved the inequality $k \geq 2 d-1$, where $k$ is the maximum number of points on the surface of a $d$-dimensional convex body, every pair of which is strictly antipodal. It has been conjectured that $k=2 d-1$, but Erdös and Füredi [2] proved the inequality $k>(1.15)^{d}$.

Another problem was originated by Grünbaum [3], who asked about the numbers of antipodal pairs and strictly antipodal pairs of points in an $n$-pointed set in $E^{d}$. Some upper bounds for these numbers were obtained by Makai and Martini [5].

In this paper we determine the greatest lower bounds for the numbers of antipodal pairs and strictly antipodal pairs of points in a finite set in $E^{d}$. In order to avoid degenerate situations, we consider the set to be the vertex-set of a convex polytope. 


\section{Main Results}

In what follows $P_{n}$ denotes a convex $d$-polytope with $n$ vertices $v_{1}, \ldots, v_{n}$ in $E^{d}$. Let $r\left(P_{n}\right)$ and $s\left(P_{n}\right)$ be, respectively, the numbers of antipodal pairs and strictly antipodal pairs of vertices in $P_{n}$.

The following result gives exact values of $r\left(P_{n}\right)$ and $s\left(P_{n}\right)$ in the case $d=2$.

Theorem 1. For a convex polygon $P_{n} \subset E^{2}$,

$$
r\left(P_{n}\right)=n+k \quad \text { and } \quad s\left(P_{n}\right)=n-k
$$

where $k(0 \leq k \leq\lfloor n / 2\rfloor)$ is the number of pairs of parallel sides in $P_{n}$.

The inequalities

$$
n \leq r\left(P_{n}\right) \leq 3 n / 2 \text { and }\lceil n / 2\rceil \leq s\left(P_{n}\right) \leq n
$$

mentioned by Grünbaum [3] for $d=2$, follow from Theorem 1 .

Theorem 2. For a convex polytope $P_{n} \subset E^{d}, n \geq d+1, d \geq 3$,

$$
r\left(P_{n}\right) \geq n-1+d(d-1) / 2
$$

The equality

$$
r\left(P_{n}\right)=n-1+d(d-1) / 2
$$

holds if and only if $P_{n}$ has $d+1$ pairwise antipodal vertices, say $v_{1}, \ldots, v_{d+1}$, and every other vertex $v_{k}$ (in the case $n>d+1$ ) is antipodal to a unique vertex $v_{i}$, $1 \leq i \leq d+1<k \leq n$.

For any vertex $v_{i}$ of $P_{n}$, denote the supporting cone of $P_{n}$ at $v_{i}$ by $C_{i}$ :

$$
C_{i}=\bigcup\left\{v_{i}+\lambda\left(P_{n}-v_{i}\right): \lambda \geq 0\right\}
$$

Theorem 3. For a convex polytope $P_{n} \subset E^{d}, n \geq d+1, d \geq 3$,

$$
s\left(P_{n}\right) \geq\lceil n / 2\rceil
$$

For an even $n$, the equality

$$
s\left(P_{n}\right)=\lceil n / 2\rceil
$$


holds if and only if $n \geq 2 d$ and the vertex-set $\left\{v_{1}, \ldots, v_{n}\right\}$ can be divided into $n / 2$ pairwise disjoint subsets of the form $\left\{v_{i}, v_{j}\right\}$ such that

$$
C_{i}-v_{i}=v_{j}-C_{j}
$$

for each of them. For an odd $n$, equality (1) holds if and only if $n \geq 4 d-1$ and some $(n-3) / 2$ pairwise disjoint subsets of the form $\left\{v_{i}, v_{j}\right\}$ can be chosen in $\left\{v_{1}, \ldots, v_{n}\right\}$ such that

$$
C_{i}-v_{i}=v_{j}-C_{j}
$$

for each of them, and the remaining three vertices, say $v_{k}, v_{l}, v_{m}$, satisfy the relation

$$
\left(C_{k}-v_{k}\right) \cap\left(C_{l}-v_{l}\right)=v_{m}-C_{m} .
$$

Observation. For every even $n \geq 2 d$ and for every odd $n \geq 4 d-1$ there is a d-polytope $P_{n} \subset E^{d}$ satisfying (1).

Note that, for a convex polytope $P_{n} \subset E^{d}$ with a small number of vertices, lower bounds for $s\left(P_{n}\right)$ are considered by Nguyên [7] and Makai and Martini [6].

It is shown later that Theorem 2 is a direct consequence of the following assertion. Let $\mathscr{M}=\left\{K_{1}, \ldots, K_{p}\right\}$ be a family of convex cones with common zero apex. Two cones $K_{i}, K_{j} \in \mathscr{M}$ are called opposite provided $K_{i} \cap\left(-K_{j}\right) \neq\{0\}$ (or $\left(-K_{i}\right) \cap K_{j} \neq\{0\}$, which is the same). Denote by $t(\mathscr{M})$ the number of all opposite pairs of cones in $\mathscr{M}$.

Theorem 4. Let $\mathscr{L}=\left\{K_{1}, \ldots, K_{n}\right\}$ be a family of closed d-dimensional convex acute polyhedral cones with a common zero apex in $E^{d}, d \geq 2$, such that

$$
\bigcup_{i=1}^{n} K_{i}=E^{d}, \quad \text { int } K_{i} \cap \text { int } K_{j}=\varnothing, \quad i \neq j
$$

and the intersection $K_{i} \cap K_{j}$ of the two cones $K_{i}, K_{j}$ is a face for both of them. Then

$$
t(\mathscr{L}) \geq n-1+d(d-1) / 2 .
$$

In the case $d=2$, the equality

$$
t(\mathscr{L})=n-1+d(d-1) / 2
$$

holds if and only if no two boundary rays of $K_{1}, \ldots, K_{n}$ are opposite, and in the case $d \geq 3$ equality (4) holds if and only if $\mathscr{L}$ satisfies the following property:

(S) There are $d+1$ pairwise opposite cones, say $K_{1}, \ldots, K_{d+1}$, and every other cone $K_{m}$ (in the case $n>d+1$ ) is opposite to a unique cone $K_{i}$, $1 \leq i \leq d+1<m \leq n$. 


\section{Proof of Theorem 4}

From now on we consider $\mathscr{L}$ to be a family of cones satisfying the hypothesis of Theorem 4. We have divided proof of this theorem into a sequence of lemmas.

For any cone $K \in \mathscr{L}$, denote by $\gamma(K)$ the number of cones in $\mathscr{L}$ opposite to $K$. It is easily seen that

$$
t(\mathscr{L})=\left(\gamma\left(K_{1}\right)+\cdots+\gamma\left(K_{n}\right)\right) / 2 .
$$

A cone $K \in \mathscr{L}$ is called special provided $\gamma(K) \geq d$.

Lemma 1. Every cone $K \in \mathscr{L}$ is opposite to at least one special cone.

Proof. Let $K$ be a cone in $\mathscr{L}$. $K$, being acute, has at least one extremal ray, say $l$. It is possible to prove (see, for instance, [8]) that $l$ lies in the boundary of at least $d-1$ cones in $\mathscr{L}$ distinct from $K$. The opposite ray $-l$ belongs to no cone in $\mathscr{L}$ containing $l$. Due to (2), $-l$ lies in a new cone $K^{\prime} \in \mathscr{L}$. Hence $K^{\prime}$ is opposite to at least $d$ cones in $\mathscr{L}$, i.e., $K^{\prime}$ is special.

Lemma 2. Every cone $K \in \mathscr{L}$ with $\gamma(K) \geq 2$ is opposite to at least two special cones.

Proof. Let $\gamma(K) \geq 2$ for a cone $K \in \mathscr{L}$. By Lemma 1 there is a special cone $K^{\prime} \in \mathscr{L}$ opposite to $K$. Since $\gamma(K) \geq 2$, we have $-K \not \subset$ int $K^{\prime}$. Hence there is an extremal ray $l$ of $-K$ which is not in the interior of $K^{\prime}$. In this case $l$ belongs to a new cone $K^{\prime \prime} \in \mathscr{L}$ and $K^{\prime \prime}$ is opposite to $K$. As in Lemma 1 , the ray $-l$, being extremal for $K$, belongs to at least $d$ cones in $\mathscr{L}$. Hence $K^{\prime \prime}$ is opposite to at least $d$ cones in $\mathscr{L}$, i.e., $K^{\prime \prime}$ is special.

Corollary 1. $\mathscr{L}$ contains at least three special cones.

Lemma 3. $\mathscr{L}$ has at least four special cones in the case $d=3$.

Proof. Assume, in order to obtain a contradiction, that $\mathscr{L}$ contains exactly three special cones, say $K_{1}, K_{2}, K_{3}$ (see Corollary 1). We distinguish two cases.

Case 1: $\operatorname{dim}\left(K_{1} \cap K_{2}\right) \leq 1$. Since $K_{1} \cap K_{2}$ is a face for both $K_{1}, K_{2}$, a plane $H$ separating $K_{1}, K_{2}$ can be chosen such that

$$
\operatorname{dim}\left(H \cap K_{1}\right) \leq 1 \text { and } \operatorname{dim}\left(H \cap K_{2}\right) \leq 1
$$

We may assume that $H \cap$ int $K_{i} \neq \varnothing$ for every cone $K_{i}$ satisfying the condition $\operatorname{dim}\left(H \cap K_{i}\right)=2$, since otherwise it is possible to move $H$ slightly in order to obtain the desired property. Let $K_{1}^{\prime}, \ldots, K_{m}^{\prime}$ be the cones in $\mathscr{L}$ whose intersection with $H$ is two-dimensional. It is easily seen that the family

$$
\mathscr{M}=\left\{H \cap K_{1}^{\prime}, \ldots, H \cap K_{m}^{\prime}\right\}
$$


satisfies the hypothesis of Theorem 4 (with $H$ instead of $E^{3}$ ). Due to Corollary 1 , there are at least three special cones, say $L_{i}=K_{i}^{\prime} \cap H, i=1,2,3$, in $\mathscr{M}$. In other words, $\gamma_{H}\left(L_{i}\right) \geq 2, i=1,2,3$, where $\gamma_{H}(L)$ means the number of cones in $\mathscr{M}$ opposite to a cone $L \in \mathscr{M}$. At least two of the cones $K_{1}^{\prime}, K_{2}^{\prime}, K_{3}^{\prime}$, say $K_{1}^{\prime}, K_{2}^{\prime}$, are different from $K_{3}$. Due to the choice of $H$, cones $K_{1}^{\prime}, K_{2}^{\prime}$ are different from $K_{1}, K_{2}$. Since $\gamma\left(K_{i}^{\prime}\right) \geq \gamma_{H}\left(L_{i}\right) \geq 2, i=1,2$, and since there are exactly three special cones in $\mathscr{L}$, each of $K_{1}^{\prime}, K_{2}^{\prime}$ is opposite to at least one of the cones $K_{1}, K_{2}$ (see Lemma 2). Hence each of $K_{1}^{\prime}, K_{2}^{\prime}$ is opposite to at least two cones in $\left\{K_{1}^{\prime}, \ldots, K_{m}^{\prime}\right\}$ and to at least one cone in $\left\{K_{1}^{\prime}, K_{2}^{\prime}\right\}$, i.e., both $K_{1}^{\prime}, K_{2}^{\prime}$ are special in $\mathscr{L}$. In this case $\mathscr{L}$ has at least five special cones, a contradiction.

Case 2: $\operatorname{dim}\left(K_{1} \cap K_{2}\right)=2$. In this case the unique hyperplane, say $H$, separating $K_{1}$ and $K_{2}$ exists. As above, let $K_{1}^{\prime}, \ldots, K_{m}^{\prime}$ be the cones in $\mathscr{L}$ whose intersection with $H$ is two-dimensional. Consider the family $\mathscr{M}=\left\{H \cap K_{1}^{\prime}, \ldots, H \cap K_{m}^{\prime}\right\}$. Unlike case 1 , some of the cones $H \cap K_{1}^{\prime}, \ldots, H \cap K_{m}^{\prime}$ may coincide. Choose in $\mathscr{M}$ the subfamily $\mathscr{F}$ of all pairwise distinct cones. As above, $\mathscr{F}$ satisfies the hypothesis of Theorem 4. By Corollary 1, in $\mathscr{F}$ a special cone of the form $K^{\prime} \cap H$ can be chosen which is different from the cones $K_{1} \cap K_{2}, K_{3} \cap H$. If $K^{\prime} \cap H$ is opposite to both cones $K_{1} \cap K_{2}, K_{3} \cap H$, then $K^{\prime}$ is opposite to $K_{1}, K_{2}, K_{3}$, i.e., $\gamma\left(K^{\prime}\right) \geq 3$. If $K^{\prime} \cap H$ is opposite to at most one of $K_{1} \cap K_{2}, K_{3} \cap H$, then, similarly to case $1, \gamma\left(K^{\prime}\right) \geq 3$. In both cases $K^{\prime}$ is a new special cone in $\mathscr{L}$, a contradiction.

Lemma 4. If for a cone $K \in \mathscr{L}$ there are $m$ cones in $\mathscr{L}$ contained in the cone $-K$, then $\gamma(K) \geq d+m$.

Proof. Assume that cones $K_{i_{1}}, \ldots, K_{i_{m}} \in \mathscr{L}$ are contained in $-K_{1}$. Denote by $H$ a $(d-1)$-subspace satisfying the condition $H \cap K_{1}=\{0\}$. (The last is possible, since $K_{1}$ is acute.) Let $H^{\prime}$ be a translate of $H$ whose intersection with $-K_{1}$ is $(d-1)$-dimensional. Put

$$
L_{0}=H^{\prime} \cap\left(-K_{1}\right), \quad L_{i}=H^{\prime} \cap K_{i}, \quad j=1, \ldots, n
$$

The set $M=L_{i_{1}} \cup \cdots \cup L_{i_{m}}$ is compact and lies in $L_{0}$. Besides $K_{i_{1}}, \ldots, K_{i_{m}}$, the hyperplane $H^{\prime}$ intersects some other cones, say $K_{p_{1}}, \ldots, K_{p_{q}}$, which have common points with $M$.

We claim that $q \geq d$. To prove this, choose a point $x$ in the relative interior rint $M$ of $M$, and let $B_{r}(x)$ be a $(d-1)$-dimensional ball of radius $r>0$ and center $x$, contained in $H^{\prime} \cap$ rint $M$. Denote by $G_{j}$ a $(d-2)$-plane in $H^{\prime}$ supporting $B_{r}(x)$ and separating $B_{r}(x)$ from $L_{p}, j=1, \ldots, q$. Let $W_{j}$ be the closed half-plane in $H^{\prime}$ determined by $G_{j}$ and containing $B_{r}(x)$. Clearly, $L_{p_{1}}, \ldots, L_{p_{q}}$ surround $M$. Hence the polyhedral set $W=W_{1} \cap \cdots \cap W_{q}$ is compact and has at most $q$ facets. Since the number of facets of a $(d-1)$-polytope is at least $d$, we have $q \geq d$.

By the choice of $K_{p_{1}}, \ldots, K_{p_{q}}$, each of these cones is opposite to $K_{1}$. Hence $\gamma\left(K_{1}\right) \geq q+m \geq d+m$. 
Lemma 5. If $\mathscr{L}$ contains $m$ special cones, then $t(\mathscr{L}) \geq m d / 2+n-m$.

Proof. We can certainly assume that $\mathscr{L}_{1}=\left\{K_{1}, \ldots, K_{m}\right\}, m \leq n$, is the family of all the special cones in $\mathscr{L}$. Put $\mathscr{L}_{2}=\mathscr{L} \backslash \mathscr{L}_{1}$ and divide $\mathscr{L}_{2}$ into subfamilies

$$
\mathscr{V}=\left\{K \in \mathscr{L}_{2}: \gamma(K)=1\right\} \quad \text { and } \quad \mathscr{W}=\left\{K \in \mathscr{L}_{2}: \gamma(K) \geq 2\right\}
$$

$\mathscr{V}$ represents the union of pairwise disjoint subfamilies $\mathscr{V}_{1}, \ldots, \mathscr{V}_{p}$ such that all members of $\mathscr{V}_{j}$ are opposite to the same cone, say $K_{i,} \in \mathscr{L}$, and cones $K_{i_{1}}, \ldots, K_{i_{p}}$ are pairwise distinct. By Lemma $2, \gamma\left(K_{i}\right) \geq d+$ card $\mathscr{V}_{j}$. Hence every cone $K_{i}$ is special.

For any cone $K \in \mathscr{L}$, denote by $\gamma^{\prime}(K)$ the number of cones in $\mathscr{L} \backslash \mathscr{V}$ opposite to $K$. By the above, $\gamma^{\prime}(K)=\gamma(K)$ if $K$ is not of the form $K_{i,}$, and $\gamma^{\prime}(K) \geq d$ if $K=K_{i,}$ for $j=1, \ldots, p$. Then

$$
\begin{aligned}
2 t(\mathscr{L})= & \sum_{i=1}^{n} \gamma\left(K_{i}\right) \\
= & \sum_{i=1}^{m} \gamma^{\prime}\left(K_{i}\right)+\sum_{j=1}^{p}\left(\gamma\left(K_{i}\right)-\gamma^{\prime}\left(K_{i}\right)+\sum\left\{\gamma(K): K \in \mathscr{V}_{j}\right\}\right) \\
& +\sum\{\gamma(K): K \in \mathscr{W}\} \\
\geq & m d+2 \sum_{j=1}^{p} \operatorname{card} \mathscr{V}_{j}+2 \operatorname{card} \mathscr{W} \\
= & m d+2(n-m) .
\end{aligned}
$$

Corollaries 2 and 3 follow from Lemmas 3 and 5.

Corollary 2. Inequality (3) holds in the case $d \leq 3$.

Corollary 3. In the case $d \geq 3$, equality (4) holds only if $\mathscr{L}$ has at most $d+1$ special cones.

Indeed, inequality

$$
n-1+d(d-1) / 2=t(\mathscr{L}) \geq m d / 2+n-m
$$

is equivalent to $d(d-2) \geq(m-1)(d-2)$.

Lemma 6. In the case $d=2$, we have $t(\mathscr{L}) \geq n$ with $t(\mathscr{L})=n$ if and only if no two boundary rays of $K_{1}, \ldots, K_{n}$ are opposite.

Proof. The inequality $t(\mathscr{L}) \geq n$ is mentioned in Corollary 2. Repeating the 
proof of Lemma 5 for $d=2$, we obtain that $t(\mathscr{L})=n$ holds if and only if

$$
\gamma\left(K_{i}\right)=2+\operatorname{card} \mathscr{V}_{j}, \quad j=1, \ldots, p,
$$

and $\gamma\left(K_{q}\right)=2$ for all $q \neq i_{1}, \ldots, i_{p}$ such that $\gamma\left(K_{q}\right) \geq 2$. A trivial verification shows that both of the last conditions hold if and only if no two boundary rays of the cones $K_{1}, \ldots, K_{n}$ are opposite.

The proof of Theorem 4 is organized by induction on $d$. The case $d=2$ is considered in Lemma 6 . Assume that the hypothesis of Theorem 4 holds for all $m \leq d-1(d \geq 3)$ and let cones $K_{1}, \ldots, K_{n}$ in $E^{d}$ satisfy the hypothesis of Theorem 4.

First we prove the first part of the assertion, namely, inequality (3).

Lemma 7. Let $\mathscr{L}$ contain a pair of nonopposite cones $K_{i}, K_{j}$ with:

(i) $\gamma\left(K_{i}\right)+\gamma\left(K_{j}\right) \geq 2 d$ in the case $d \geq 3$.

(ii) $\gamma\left(K_{i}\right)+\gamma\left(K_{j}\right) \geq 2 d-1$ in the case $d \geq 4$.

Then $t(\mathscr{L})>n-1+d(d-1) / 2$.

Proof. Since $K_{i}, K_{j}$ are nonopposite (i.e., $\left.K_{i} \cap\left(-K_{j}\right)=\{0\}\right)$, there is a $(d-1)$ subspace $H$ separating $K_{i}$ and $-K_{j}$ such that $H \cap\left(K_{i} \cup K_{j}\right)=\{0\}$. Without loss of generality, we may put $i=1, j=2$.

It can be assumed that $H \cap$ int $K_{i} \neq \varnothing$ for every cone $K_{i}$ satisfying $H \cap K_{i} \neq$ $\{0\}$, since otherwise it is possible to move $H$ slightly in order to obtain the desired property. Let $K_{3}, \ldots, K_{l}$ be the cones in $\mathscr{L}$ whose interiors intersect $H$. It is easily seen that the family $\mathscr{M}=\left\{H \cap K_{3}, \ldots, H \cap K_{l}\right\}$ satisfies the hypothesis of Theorem 4 such that (2) holds (with $H$ instead of $E^{d}$ ). By inductive hypothesis,

$$
t(\mathscr{M}) \geq l-3+(d-1)(d-2) / 2 .
$$

Put $\mathscr{L}_{1}=\left\{K_{3}, \ldots, K_{l}\right\}$. Denote by $\mathscr{L}_{2}$ the family of all special cones in $\mathscr{L} \backslash\left(\mathscr{L}_{1} \cup\left\{K_{1}, K_{2}\right\}\right)$, and let

$$
\mathscr{L}_{3}=\mathscr{L} \backslash\left(\mathscr{L}_{1} \cup \mathscr{L}_{2} \cup\left\{K_{1}, K_{2}\right\}\right)
$$

Then

$$
\sum_{i=1}^{n} \gamma\left(K_{i}\right)=\gamma\left(K_{1}\right)+\gamma\left(K_{2}\right)+\sum_{i=1}^{3}\left(\sum\left\{\gamma(K): K \in \mathscr{L}_{i}\right\}\right)
$$

As in Lemma 5 , divide $\mathscr{L}_{3}$ into the subfamilies

$$
\mathscr{V}=\left\{K \in \mathscr{L}_{3}: \gamma(K)=1\right\} \text { and } \mathscr{W}=\left\{K \in \mathscr{L}_{3}: \gamma(K) \geq 2\right\}
$$


and introduce the function $\gamma^{\prime}(K)$. Then

$$
\begin{aligned}
2 t(\mathscr{L})= & \sum_{i=1}^{n} \gamma\left(K_{i}\right) \\
= & \gamma^{\prime}\left(K_{1}\right)+\gamma^{\prime}\left(K_{2}\right)+\sum\left\{\gamma^{\prime}(K): K \in \mathscr{L}_{1}\right\}+\sum\left\{\gamma^{\prime}(K): K \in \mathscr{L}_{2}\right\} \\
& +\sum_{j=1}^{p}\left(\gamma\left(K_{i}\right)-\gamma^{\prime}\left(K_{i}\right)+\operatorname{card} \mathscr{V}_{j}\right)+\sum\left\{\gamma\left(K_{i}\right): K \in \mathscr{W}\right\} \\
\geq & (2 d-1)+2 t\left(\mathscr{L}_{1}\right)+d \cdot \operatorname{card} \mathscr{L}_{2}+2 \text { card } \mathscr{V}+2 \text { card } \mathscr{W} \\
= & (2 d-1)+2 t\left(\mathscr{L}_{1}\right)+d \cdot \operatorname{card} \mathscr{L}_{2}+2 \text { card } \mathscr{L}_{3} .
\end{aligned}
$$

Due to Lemma $2, K_{1}$ is opposite to at least two special cones $K_{1}^{\prime}, K_{1}^{\prime \prime} \in \mathscr{L}$, and $K_{2}$ is opposite to at least two special cones $K_{2}^{\prime}, K_{2}^{\prime \prime} \in \mathscr{L}$. Since $K_{1} \backslash\{0\}$ and $K_{2} \backslash\{0\}$ lie in the same open half-space determined by $H$, cones $K_{1}$ and $K_{2}$ are nonopposite and any of them differs from each of $K_{1}^{\prime}, K_{1}^{\prime \prime}, K_{2}^{\prime}, K_{2}^{\prime \prime}$.

We consider separately the following three cases.

Case 1. If $K_{1}^{\prime}, K_{1}^{\prime \prime}, K_{2}^{\prime}, K_{2}^{\prime \prime}$ are pairwise distinct, and $p(0 \leq p \leq 4)$ of them belong to $\mathscr{L}_{1}$, then

$$
2 t\left(\mathscr{L}_{1}\right) \geq 2 t(\mathscr{M})+p, \quad \text { card } \mathscr{L}_{2} \geq 4-p
$$

and

$$
\begin{aligned}
2 t(\mathscr{L}) & \geq 2 d-1+2 t(\mathscr{M})+p+d(4-p)+2(n-l-4+p) \\
& \geq 2 n-1+d(d-1) .
\end{aligned}
$$

Case 2. Let, for example, $K_{1}^{\prime}=K_{2}^{\prime}$ and $K_{1}^{\prime}, K_{1}^{\prime \prime}, K_{2}^{\prime \prime}$ be pairwise distinct. Since $\mathscr{L}$ has at least five special cones, we have $t(\mathscr{L})>n+2$ in the case $d=3$ (see Corollary 2). Suppose that $d \geq 4$. If all cones $K_{1}^{\prime}, K_{1}^{\prime \prime}, K_{2}^{\prime \prime}$ belong to $\mathscr{L}_{1}$, then

$$
2 t(\mathscr{L}) \geq 2 d-1+2 t(\mathscr{M})+4+2(n-l) \geq 2 n-1+d(d-1) .
$$

Assume that only some $q(0 \leq q \leq 2)$ of $K_{1}^{\prime}, K_{1}^{\prime \prime}, K_{2}^{\prime \prime}$ belong to $\mathscr{L}_{1}$. Then $2 t\left(\mathscr{L}_{1}\right) \geq 2 t(\mathscr{M})+q$ and

$$
\begin{aligned}
2 t(\mathscr{L}) & \geq 2 d-1+2 t(\mathscr{M})+q+d(3-q)+2(n-l-3+q) \\
& \geq 2 n-1+d(d-1) .
\end{aligned}
$$

Case 3. Let $K_{1}^{\prime}=K_{2}^{\prime}$ and $K_{1}^{\prime \prime}=K_{2}^{\prime \prime}$. As above,

$$
\begin{aligned}
2 t(\mathscr{L}) & \geq 2 d-1+2 t(\mathscr{M})+q+d(2-v)+2(n-l-2+v) \\
& \geq 2 n-1+d(d-1)+(d-4)(2-v),
\end{aligned}
$$


where $v(0 \leq v \leq 2)$ is the number of cones in $\left\{K_{1}^{\prime}, K_{1}^{\prime \prime}\right\}$ contained in $\mathscr{L}_{1}$. We have

$$
2 n-1+d(d-1)+(d-4)(2-v) \geq 2 n-1+d(d-1)
$$

if $d \geq 4$ or $d=3$ and $v=2$. Hence it remains to consider the case $d=3$ and $v \leq 1$. By the hypothesis, $\gamma^{\prime}\left(K_{1}\right)+\gamma^{\prime}\left(K_{2}\right) \geq 2 d$. If $v=1$, then

$$
2 t(\mathscr{L}) \geq 2 d+2 t(\mathscr{M})+2+d+2(n-l-1) \geq 2 n-1+d(d-1) .
$$

Let $v=0$, i.e., $K_{1}^{\prime}, K_{1}^{\prime \prime} \in \mathscr{L}_{2}$. Due to Corollary 2, it is sufficient to consider the case when $K_{1}, K_{2}, K_{1}^{\prime}, K_{1}^{\prime \prime}$ are the only special cones in $\mathscr{L}$. Since $\mathscr{M}$ satisfies the hypothesis of Theorem 4 (with $H$ instead of $E^{d}$ ), it contains at least three special cones (see Corollary 2). Hence there are cones $L_{1}, L_{2}, L_{3} \in \mathscr{L}_{1}$ with $\gamma\left(L_{i}\right) \geq 2$ for all $i=1,2,3$. Due to Lemma 2, every $L_{i}$ is opposite to two special cones in $\mathscr{L}$. By the assumption, these cones are of the form $K_{1}, K_{2}, K_{1}^{\prime}, K_{1}^{\prime \prime}$. In this case $2 t\left(\mathscr{L}_{1}\right) \geq 2 t(\mathscr{M})+6$ and

$$
2 t(\mathscr{L}) \geq 2 d+2 t(\mathscr{M})+6+2 d+2(n-l-2)>2 n+d(d-1) .
$$

In all the cases above, $t(\mathscr{L})>n-1+d(d-1) / 2$.

Lemma 8. Let $d \geq 4$ and let $\mathscr{L}$ be such that every two cones $K_{i}, K_{j} \in \mathscr{L}$ are opposite if $\gamma\left(K_{i}\right)+\gamma\left(K_{j}\right) \geq 2 d-1$. Then (3) holds.

Proof. Due to Corollary 2, there are at least three special cones, say $K_{1}, K_{2}$, $K_{3} \in \mathscr{L}$. We distinguish two cases.

Case 1: $\operatorname{dim}\left(K_{1} \cap K_{2}\right) \leq d-2$. In this case a hyperplane $H$ separating $K_{1}, K_{2}$ can be chosen such that

$$
\operatorname{dim}\left(H \cap K_{1}\right) \leq d-2 \text { and } \operatorname{dim}\left(H \cap K_{2}\right) \leq d-2
$$

Let $\mathscr{H}$ be the family of cones in $\mathscr{L}$ intersected by $H$. If $K_{3}$ were not in $\mathscr{H}$, then $K_{3}$ would not be antipodal to one of $K_{1}, K_{2}$. Hence $K_{3} \in \mathscr{H}$. Choose in $\mathscr{H}$ the subfamily $\mathscr{F}$ of all pairwise distinct cones whose intersection with $H$ is $(d-1)$ dimensional. As above, $K_{3} \in \mathscr{F}$. Without loss of generality, it can be assumed that $\mathscr{F}=\mathscr{H}=\left\{K_{3}, \ldots, K_{l}\right\}$.

By inductive hypothesis (see Lemma 7),

$$
t(\mathscr{H}) \geq l-3+(d-1)(d-2) / 2
$$

Due to Corollary 1 , there are at least three special cones, say $L_{i}=K_{i}^{\prime} \cap H, i=1$, 2,3 , in the family $\mathscr{H}=\left\{H \cap K_{3}, \ldots, H \cap K_{l}\right\}$. In other words, $\gamma_{H}\left(L_{i}\right) \geq d-1$, $i=1,2,3$, where $\gamma_{H}(L)$ means the number of cones in $\mathscr{M}$ opposite to $L$. Since 
$K_{3} \in \mathscr{H}$, at least two of the cones $K_{1}^{\prime}, K_{2}^{\prime}, K_{3}^{\prime}$, say $K_{1}^{\prime}, K_{2}^{\prime}$, are distinct from $K_{3}$. Due to

$$
\gamma\left(K_{i}^{\prime}\right) \geq \gamma_{H}\left(L_{i}\right) \geq d-1, \quad i=1,2
$$

each of $K_{1}^{\prime}, K_{2}^{\prime}$ is opposite to both $K_{1}, K_{2}$. Hence

$$
2 t(\mathscr{L}) \geq 2 d+2 t(\mathscr{H})+4+2(n-l) \geq 2 n+d(d-1) .
$$

Case 2: $\operatorname{dim}\left(K_{1} \cap K_{2}\right)=d-1$. Let $H$ be the hyperplane separating $K_{1}, K_{2}$. As above, assume $\mathscr{H}=\left\{K_{2}, \ldots, K_{l}\right\}$ is the family of all cones in $\mathscr{L} \backslash\left\{K_{1}\right\}$ whose intersection with $H$ is $(d-1)$-dimensional. A special cone of the form $K^{\prime} \cap H \in \mathscr{M}$ distinct from the cones $K_{1} \cap K_{2}, K_{3} \cap H$ can be chosen. Since $\gamma\left(K^{\prime}\right) \geq d-1, K^{\prime}$ is antipodal to $K_{1}$. By the antipodality of $K_{1}$ and $K_{3}$,

$$
2 t(\mathscr{L}) \geq 2 d+2 t(\mathscr{H})+2+2(n-l) \geq 2 n-2+d(d-1) .
$$

Summing up the assertions of Corollary 2 and Lemmas 7 and 8 , we obtain that inequality (3) holds for all $d \geq 3$. To finish the proof of Theorem 4 , it remains to consider the conditions under which equality (4) holds when $d \geq 3$.

Lemma 9. In the case $d \geq 3$, equality (4) holds if and only if $\mathscr{L}$ has property (S).

Proof. First we show that $\mathscr{L}$ has $d+1$ special cones. The case $d=3$ follows from Lemma 3 and Corollary 3. Let $d \geq 4$. Choose in $\mathscr{L}$ two special cones, say $K_{1}, K_{2}$, and let $H$ be a hyperplane separating $K_{1}, K_{2}$. As in Lemma 8, consider the family $\mathscr{H}$, say $\left\{K_{3}, \ldots, K_{l}\right\}$, of cones in $\mathscr{L}$ whose intersection with $H$ is $(d-1)$-dimensional. Analysis of the proof of Lemma 8 shows that (4) holds only if $\operatorname{dim}\left(K_{1} \cap K_{2}\right)=d-1$. Then

$$
\begin{aligned}
2 n-2+d(d-1) & =2 t(\mathscr{L}) \geq 2 d+2 t(\mathscr{H})+2+2(n-l) \\
& \geq 2 n-2+d(d-1) .
\end{aligned}
$$

Hence

$$
t(\mathscr{M})=l-3+(d-1)(d-2) / 2 .
$$

By inductive hypothesis, $\mathscr{M}$ has $d$ special cones. Since $K_{1} \cap K_{2}$ belongs to $\mathscr{M}$, at least $d-1$ special cones in $\mathscr{M}$ of the form $K_{1}^{\prime} \cap H, \ldots, K_{d}^{\prime} \cap H$ are different from $K_{1} \cap K_{2}$, i.e., they satisfy $\gamma_{H}\left(K_{i}^{\prime}\right) \geq d$, where $\gamma_{H}\left(K_{i}^{\prime}\right)$ means the number of cones in $\mathscr{M}$ opposite to $K_{i}^{\prime} \cap H$. Due to Lemma 7, each of $K_{1}^{\prime}, \ldots, K_{d}^{\prime}$ is opposite to both $K_{1}, K_{2}$. Therefore we have found $d+1$ special cones in $\mathscr{L}$.

Since property (S) trivially implies (4), it remains to prove that (4) implies (S). From the proof of Lemma 5 it follows that $\gamma(K)=d$ for every special cone $K \in \mathscr{L}$ 
and the number of special cones in $\mathscr{L}$ is at most $d+1$. By the above, $\mathscr{L}$ has exactly $d+1$ special cones. Due to Lemma 7, each of the two special cones in $\mathscr{L}$ are opposite. Hence all special cones in $\mathscr{L}$ generate $d(d+1) / 2$ pairs of opposite cones. In this situation, every nonspecial cone $K \in \mathscr{L}$ determines exactly $\gamma(K)$ new pairs of opposite cones. Since the number of these new pairs equals $n-d-1$ (see the proof of Lemma 5), the last is possible only if $\mathscr{W}=\varnothing$, i.e., if $\mathscr{L}$ has property (S).

\section{Proofs of Theorems 1-3}

Proof of Theorem 1. Let $v_{1}, \ldots, v_{n}$ be all the vertices of $P_{n}$ given in the clockwise order. Two pairs of vertices in $P_{n}$ of the form

$$
\left\{v_{i}, v_{j+1}\right\},\left\{v_{i+1}, v_{j}\right\}, \quad i \neq j+1, \quad j \neq i+1,
$$

are called adjacent. It is easy to check that this relation of adjacency divides the family of all pairs of vertices in $P_{n}$ into $n$ pairwise disjoint classes, say $\mathscr{A}_{1}, \ldots, \mathscr{A}_{n}$. Simple geometric considerations show that:

1. Every class $\mathscr{A}_{\mathrm{k}}$ contains at least one but at most two antipodal pairs of vertices in $P_{n}$.

2. A class $\mathscr{A}_{k}$ contains two antipodal pairs, say $\left\{v_{i}, v_{j+1}\right\},\left\{v_{i+1}, v_{j}\right\}$, if and only if the sides $\left[v_{i}, v_{i+1}\right],\left[v_{j}, v_{j+1}\right]$ of $P_{n}$ are parallel.

Similarly, every class $\mathscr{A}_{k}$ contains at most one strictly antipodal pair of vertices in $P_{n}$ and $\mathscr{A}_{k}$ contains no strictly antipodal pair if and only if there are two adjacent pairs, say $\left\{v_{i}, v_{j+1}\right\},\left\{v_{i+1}, v_{j}\right\}$, in $\mathscr{A}_{k}$ such that the sides $\left[v_{i}, v_{i+1}\right],\left[v_{j}, v_{j+1}\right]$ are parallel. Now the assertion of Theorem 1 readily follows.

Proof of Theorem 2. Let $P_{n} \subset E^{d}$ be a convex polytope with $n$ vertices. For every vertex $v_{i}$ of $P_{n}$, denote by $K_{i}^{\prime}$ the cone generated by all extremal normals to $P_{n}$ at $v_{i}$. Consider the cones

$$
K_{i}=K_{i}^{\prime}-v_{i}, \quad i=1, \ldots, n
$$

We see at once that the family $\mathscr{L}=\left\{K_{1}, \ldots, K_{n}\right\}$ satisfies the hypothesis of Theorem 4. Moreover, vertices $v_{i}, v_{j}$ of $P_{n}$ are antipodal if and only if the respective cones $K_{i}, K_{j}$ are opposite. Now Theorem 2 trivially follows from Theorem 4.

Proof of Theorem 3. The first assertion of Theorem 3 is trivial. Indeed, every vertex $v_{i}$ in $P_{n}$ belongs to at least one strictly antipodal pair of vertices (see, for instance, [8]). Hence $s\left(P_{n}\right) \geq\lceil n / 2\rceil$.

Below we determine the conditions under which (1) holds.

1. Let $n=2 k$. It is proved in [8] that $s(B) \geq d$ for every convex body $B \subset E^{d}$ with $s(B)=d$ if and only if $B$ is a cross-polytope. Hence (1) holds for $n \geq 2 d$ only. 
If $s\left(P_{2 k}\right)=k$, then every vertex of $P_{2 k}$ belongs to exactly one strictly antipodal pair, i.e., the vertices of $P_{2 k}$ can be uniquely divided into disjoint pairs, say $\left\{v_{i}, v_{i+k}\right\}, i=1, \ldots, k$, describing all antipodal pairs of vertices in $P_{2 k}$.

Assume, in order to obtain a contradiction, that

$$
C_{i}-v_{i} \not \subset v_{i+k}-C_{i+k}
$$

for an index $i=1, \ldots, k$. Then there is an edge $\left[v_{i}, v_{j}\right]$ of $P_{2 k}$ such that $v_{j}$ is not in the cone $v_{i}+v_{i+k}-C_{i+k}$. Denote by $H$ a hyperplane passing through $v_{i}$ and strictly separating $v_{j}$ from $v_{i}+v_{i+k}-C_{i+k}$. If $H^{\prime}$ is the hyperplane containing $v_{i+k}$ and parallel to $H$, then $H^{\prime} \cap P_{2 k}=\left\{v_{i+k}\right\}$. The last relation implies that $\left\{v_{j}, v_{i+k}\right\}$ is a new pair of strictly antipodal vertices different from $\left\{v_{i}, v_{i+k}\right\}$. The contradiction obtained shows that $C_{i}-v_{i} \subset v_{i+k}-C_{i-k}$. Similarly, $v_{i+k}-C_{i+k} \subset C_{i}-v_{i}$.

Conversely, if the vertex-set $v_{1}, \ldots, v_{2 k}$ of $P_{2 k}$ is divided into disjoint pairs, say $\left\{v_{i}, v_{i+k}\right\}, i=1, \ldots, k$, such that

$$
C_{i}-v_{i}=v_{i+k}-C_{i+k} \quad \text { for all } i=1, \ldots, k
$$

then, clearly, $\left\{v_{i}, v_{i+k}\right\}, i=1, \ldots, k$, describe all pairs of strictly antipodal vertices in $P_{2 k}$.

2 . Let $n=2 k+1$. The relation $s\left(P_{2 k+1}\right)=k+1$ is possible if and only if exactly one of the vertices of $P_{2 k+1}$, say $v_{1}$, belongs to two different strictly antipodal pairs and every other vertex belongs to exactly one such a pair. Assume, without loss of generality, that pairs

$$
\left\{v_{1}, v_{2}\right\},\left\{v_{1}, v_{3}\right\},\left\{v_{i}, v_{i+k-1}\right\}, \quad i=4, \ldots, k+2
$$

determine all strictly antipodal pairs of vertices in $P_{2 k+1}$. By analogy to condition 1 , we have

$$
\left.\begin{array}{rl}
v_{1}-C_{1} & =\left(C_{2}-v_{2}\right) \cap\left(C_{3}-v_{3}\right), \\
C_{i}-v_{i} & =v_{i+k-1}-C_{i+k-1}, \quad i=4, \ldots, k+2 .
\end{array}\right\}
$$

Conversely, if the vertices $v_{1}, \ldots, v_{2 k}$ of $P_{2 k}$ are divided into subsets, say (5), such that (6) holds, then (5) describes all strictly antipodal pairs of vertices in $P_{2 k}$.

It remains to prove that relation $s\left(P_{2 k+1}\right)=k+1$ is possible for $2 k+1 \geq$ $4 d-1$ only. Let $P_{2 k+1}$ be a polytope with $2 k+1$ vertices, satisfying $s\left(P_{2 k+1}\right)=$ $k+1$. By the above, there are three vertices, say $v_{1}, v_{2}, v_{3}$, such that (6) holds. In this case the line segment $\left[v_{2}, v_{3}\right]$ is an edge of $P_{2 k+1}$. From (6) it follows that the centrally symmetric convex polytope $Q=P_{2 k+1}-P_{2 k+1}$ has $2 k+2$ vertices. Since $P_{2 k+1}$ is not centrally symmetric, $Q$ is reducible (i.e., by definition it can be represented as $Q=P-P$ for a nonsymmetric convex polytope $P$ ). Due to Yost [9], any centrally symmetric reducible polytope has at least $4 d$ vertices. In the case of $Q$, we have $2 k+2 \geq 4 d$, i.e., $2 k+1 \geq 4 d-1$. 
Proof of the Observation. Let $n=2 k(\geq 2 d)$ and let $P_{n} \subset E^{d}$ be a convex $d$-polytope symmetric with respect to the origin 0 . If $v_{1}, \ldots, v_{2 k}$ are the vertices of $P_{n}$ such that $v_{i+k}=-v_{i}$ for all $i=1, \ldots, k$, then $\left\{v_{i}, v_{l+k}\right\}, i=1, \ldots, k$, describe all strictly antipodal pairs of vertices in $P_{n}$. Hence $s\left(P_{n}\right)=\lfloor n / 2\rfloor$.

Now we show that, for every natural number $m=2 k+1 \geq 4 d-1$, a $d$ polytope $P_{2 k+1} \subset E^{d}$ with $s\left(P_{2 k+1}\right)=k+1$ exists.

For this purpose, choose in $E^{d}$ a convex polytope $B$ with center 0 and $2 k-2 d+2(\geq 2 d)$ vertices $v_{1}, \ldots, v_{2 k-2 d+2}$, having a pair of symmetric facets of the form $S=\operatorname{conv}\left(v_{1}, \ldots, v_{d}\right), T=-S$. Without loss of generality, we can put $v_{k-d+i+1}=-v_{i}$ for all $i=1, \ldots, k-d+1$. Choose a point $v_{0} \notin B$ sufficiently close to the center of $S$ such that the polytope $Q=\operatorname{conv}\left(v_{0} \cup-v_{0} \cup B\right.$ ) has new (relative to $B$ ) facets

$$
S_{i}=\operatorname{conv}\left(v_{1}, \ldots, v_{i-1}, v_{0}, v_{i+1}, \ldots, v_{d}\right), \quad T_{i}=-S_{i}, \quad i=1, \ldots, d .
$$

Denote by $H$ a hyperplane satisfying the conditions:

1. $v_{1} \in H$.

2. $H$ strictly separates $v_{0}$ from $B$.

3. $H \cap Q$ is a $(d-1)$-simplex of the form $\operatorname{conv}\left(v_{1}, w_{2}, \ldots, w_{d}\right)$, where $\left.w_{i} \in\right] v_{0}, v_{i}[$, $i=2, \ldots, d$.

Similarly, let $G$ be a hyperplane strictly scparating $-v_{0}$ and $B$ such that $G \cap Q$ is a $(d-1)$-simplex of the form $\operatorname{conv}\left(z_{1}, \ldots, z_{d}\right)$, where $\left.z_{i} \in\right]-v_{0},-v_{i}[, i=1, \ldots, d$.

Denote by $P$ that part of $Q$ which lies in the closed strip between $H$ and $G$. It follows easily that $P$ is a convex $d$-polytope with $2 k+1$ vertices, namely,

$$
v_{1}, \ldots, v_{2 k-2 d+2}, w_{2}, \ldots, w_{d}, z_{1}, \ldots, z_{d}
$$

and

$$
\left\{v_{i},-v_{i}\right\}, \quad i=1, \ldots, k-d+1, \quad\left\{w_{i}, z_{i}\right\}, \quad i=2, \ldots, d, \quad\left\{v_{1}, z_{1}\right\}
$$

describe all $k-d+1+d-1+1=k+1$ strictly antipodal pairs of vertices in $P$. Hence $s(P)=k+1$.

\section{Acknowledgment}

The authors thank the referees for their helpful comments on an earlier draft of the paper.

\section{References}

1. L. Danzer, B. Grünbaum, Über zwei Probleme bezüglich konvexer Körper von P. Erdös und von V. L. Klee, Math. Z. 79 (1962), 95-99. 
2. P. Erdös, Z. Füredi, The greatest angle among $n$ points in the $d$-dimensional euclidean space, $A n n$. Discrete Math. 17 (1983), 275-283.

3. B. Grünbaum, Strictly antipodal sets, Israel J. Math. 1 (1963), 5-10.

4. V. Klee. Unsolved problems in intuitive geometry, Mimeographed notes, Seattle, 1960.

5. E. Makai, Jr., H. Martini, On the number of antipodal or strictly antipodal pairs of points in finite subsets of $R^{d}$, in: Applied Geometry and Discrete Mathematics. The V. Klee Festschrift, DIMACS Series in Discrete Mathematics and Theoretic Computer Science, Vol. 4, 1991, pp. 457-470.

6. E. Makai, Jr., H. Martini, On the number of antipodal or strictly antipodal pairs of points in finite subsets of $R^{d}$. II, Preprint No. 7/1992, Mathematical Institute of the Hungarian Academy of Science, 1992, $24 \mathrm{pp}$.

7. M. N. Nguyên, Lower estimate of the number of exposed diameters of a convex polytope with a small number of vertices, Manuscript, Kishinev State University, Kishinev, 1990, 27 pp. (Deposited in Moldavian NIINTI 22.01.90, No. 1154-M90, in Russian.)

8. V. Soltan, M. N. Nguyên, Lower bounds for the number of extremal and exposed diameters of a convex body, Studia Sci. Math. Hungar, 28 (1993), 95-100.

9. D. Yost, Irreducible convex sets, Mathematika 38 (1991), 134-155.

Received June 10, 1992, and in revised form May 14, 1993. 